\title{
ADAPTIVE LEARNING IMPLEMENTATION - A COGNITIVE DESCRIPTION EXPERIMENT FOR FIRST YEAR ENGINEERING STUDENTS AT A DISTANCE EDUCATION UNIVERSITY
}

\author{
Ngaka Mosia, Unisa, South Africa
}

\begin{abstract}
A study was performed on a first year industrial engineering statistics course to improve the statistics pass rate. Statistics is a requisite for other engineering courses. The pass rate for the statistic course was below 50\%. The primary purpose is to enable learners to build a capacity to comprehend module content and establish a deeper level of learning that will enable learners to achieve goals and objectives of T\&L lessons. An intervention program was instructionally designed to develop a personalized and differentiated learning process that breaks down lessons into lower and basic components, for struggling learners, and improves lessons to a complex high level and challenging activities for excelling students. Forty students were considered for the study. Moore's theory of transactional distance was used as a theoretical framework. The data consisted of exam and assignment scores. A quantitative method was used to analyse the data. Hypothesis testing suggests that the intervention program is significant. The overall pass rates improved by $25 \%$.
\end{abstract}

Keywords: ODeL; Scaffolding; Teaching \& Learning; Learner support.

\section{Introduction}

There is evidence that blended classroom models can be effective only when the online elements are active rather than passive, according to a 2010 department of education report, undergraduate students in blended classroom settings had better assessment outcomes than purely online or face-to-face classes. The reason being that blended courses in which the students are spending their time online solving problems, moving through the material at their own pace, and spending half of class time in the online component of the class, have more positive learning impacts than both face-to-face only and purely online only (Peters et al., 2014). 
Mosia, N.

Adaptive Learning Implementation - A Cognitive Description Experiment for First Year Engineering Students at a Distance Education University

Experience with content comprehension struggles of a first year module students has led to the development of an idea that student need a differentiated approach and different learning techniques, offering the best from both components of a blended course. It is apparent that for engineering students pacing is needed and learning must be possible on more platforms than just on the LMS. Therefore, a T\&L approach that provides a better control to the learner and is supported with online resources is indispensable (Peters, 1994). Thus, an active technology integrated T\&L approach is critical for throughput improvement.

Learning may mean acquisition of knowledge (what knowledge?) And/or deepening of understanding (of what?) And/or acquisition and improvement of both technical and interpersonal skills (which skills?) and/or development of desired attitudes and values (which attitudes and values, and desired by whom?) Understanding, skills attitudes, and values are all highly subjective constructs. Studies have shown that the more students work in cooperative learning groups the more they learn, the better they understand what they are learning, the easier it is for them to remember what they learn (Johnson et al., 1998ac). Springer et al. (1999) meta-analysed the research for college-level science, engineering and technology and found significant effects on students' persistence and achievement in these fields and positive attitudes toward their education.

Therefore, a system with the ability to adapt intelligently to goal, tasks, interests and other features of individuals and groups of users, is an ideal engineering technology education medium. In an ODeL institution, access is given to all potential students with varying grade 12 scores and therefore different competencies of the basic engineering modules such as mathematics and science. Some student have poor grades, while others have very good grades, thus in the teaching and learning process, It is not more possible to treat all students in the proliferating range of e-learning users with very different prior knowledge of the domain, backgrounds, learning styles, interests and preferences, with the "one size fits all" approach. Therefore, adaptation of the learning process and assessment is indispensable.

The power of this innovation is in matching the educational content and the complexity or simplicity of the material, with the learning style of the learners and giving consideration to the underlying pedagogical principles. The effect of the combination of strategies is observed and comprehended by analysing the assignment scores of learners and therefore, making a decision to adapt learning content by increasing the complexity of the problems or simplifying content. Since the learning styles are not static (Siadaty \& Taghiyareh, 2007), assessment, alignment and adaptation is done continuously throughout the learning period i.e. semester or year. 
Mosia, N.

Adaptive Learning Implementation - A Cognitive Description Experiment for First Year Engineering

Students at a Distance Education University

\section{Theoretical Framework}

Introduced in the early 1970s, is the theory of transactional distance by Michael Moore. Moore recognized the limitation of the structure of the independent learning package by including dialogue as a second variable. Moore's theory of transactional distance is intuitively appealing and moves the field toward the realization of a pedagogical theory. According to Moore (1991), transactional distance is pedagogical, not geographic, and necessitates "special organizations and teaching procedures" (p.3) composed of two variables (clusters, dimensions?), i.e. structure and dialogue (Moore, 1991; Moore \& Kearsley, 1996). Structure reflects the course's design and is largely a function of the teaching organization and communications media employed.

On the other hand, dialogue is also associated with the medium of communication and may include either real two-way communication or Holmberg's internal didactic conversation. In Moore's theory, the most distant program has low dialogue and low structure while the least distant has high dialogue and high structure (Moore, 1993). The innovation applied to improve student performance deals with Moore's pedagogical variables of transactional distance. The innovation works on structure as it relates to course design and communication media employed. It is also deals with dialogue, for the engagement between student and instructor is bound to increase.

\section{Approach}

The study is focused on first year engineering student, who have taken engineering statistics for the semester. Analysis is done on the results of the first assignment to measure the impact of the innovation on student performance. Adequate supports is provided for students with different learning styles. Ally and Fahy (2002) found that students with different learning styles have different preferences for support. The difficulty level of the material is set to match the cognitive level of the learner, so that the learner can both attend to and relate to the material. Cognitive description is applied on the result and student are grouped according to their performance. Poor performing students are placed on a pacing and an instructional scaffolding teaching program and excelling students are placed on an adaptive learning and gradual release of responsibility program.

\section{The experiment}

Applied techniques for mapping this approach into practice are: providing a pre-test, breaking off the learning material into small pieces, sequencing the material from easy to more difficult and providing links for further exploration to fulfil this characteristic of sensation seeking learners. This approach is implemented by applying methods such as providing the summary and outline of the content, chunking the educational material into 


\section{Mosia, N.}

\section{Adaptive Learning Implementation - A Cognitive Description Experiment for First Year Engineering}

Students at a Distance Education University

meaningful groups and giving the learners the opportunity to revisit topics to strengthen their retention. In order to evaluate the proposed framework experimentally, an investigated hypothesis was that: Matching the educational contents with the learning style of the learners along with considering the underlying pedagogical principles, affects the performance of the students in terms of learning outcomes.

Table 1:

\begin{tabular}{|c|c|c|}
\hline Research Question & Hypothesis & Prediction \\
\hline $\begin{array}{l}\text { Does, the matching of } \\
\text { educational contents with } \\
\text { learning styles of learners } \\
\text { and underlying pedagogical } \\
\text { principles, affect learners } \\
\text { performance outcomes? }\end{array}$ & $\begin{array}{l}\text { Matching the educational } \\
\text { contents with the learning style of } \\
\text { the learners along with } \\
\text { considering the underlying } \\
\text { pedagogical principles, affects } \\
\text { the performance of the students } \\
\text { in terms of learning outcomes. }\end{array}$ & $\begin{array}{l}\text { If I match module contents } \\
\text { with the learning style of the } \\
\text { learners along with } \\
\text { considering the underlying } \\
\text { pedagogical principles, } \\
\text { learner's performance } \\
\text { outcomes will improve. }\end{array}$ \\
\hline
\end{tabular}

The experimental group were provided with the personalized contents based on their learning styles whilst the other group was given the non-matched contents, originally designed for that course. The limitations of the study are that (a) since learning styles are not static, initiating the T\&L framework once (per group) when learners first use the system, might not be adequate enough. (b) It is possible that learners are not assessed as effectively as they might have been. (c) The lack of professional skills in psychology limits the application of LSP to increase self-awareness and metacognition of learners.

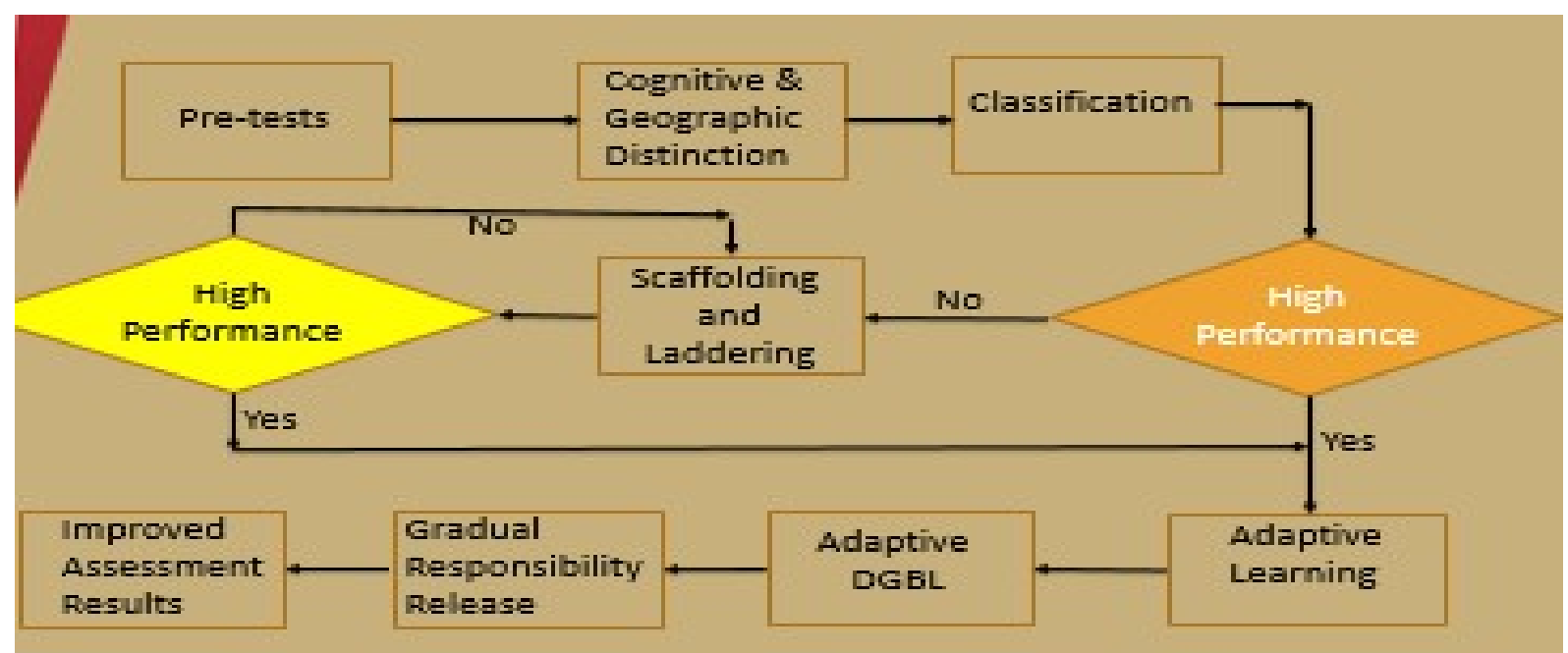

Figure 1.

Application of teaching technologies, learning objects and instructional support media tools to enable learning possibilities, when-ever, where-ever and on what-ever device student have access to at a point in time, is key to the innovation employed. This strategy consists of a representation of learning materials that the learner can engage and includes a set of domain concepts such as facts, lessons and activities organized to form a kind of a 


\section{Mosia, N.}

Adaptive Learning Implementation - A Cognitive Description Experiment for First Year Engineering Students at a Distance Education University

semantic network as stipulated by Siadaty and Taghiyareh (2007). At this stage numerous instructional support material such as OERs, LO, MOOCs from other institutions, are used.

\section{Results}

Results of the impact of the innovation on student's performance are displayed below. There is a general increase in pass rate for the years 2015 to 2018. These results imply that the innovation has a positive impact on the combined learner's performance. Note that these results represent both excellent students and students who were struggling in the beginning of the semester.
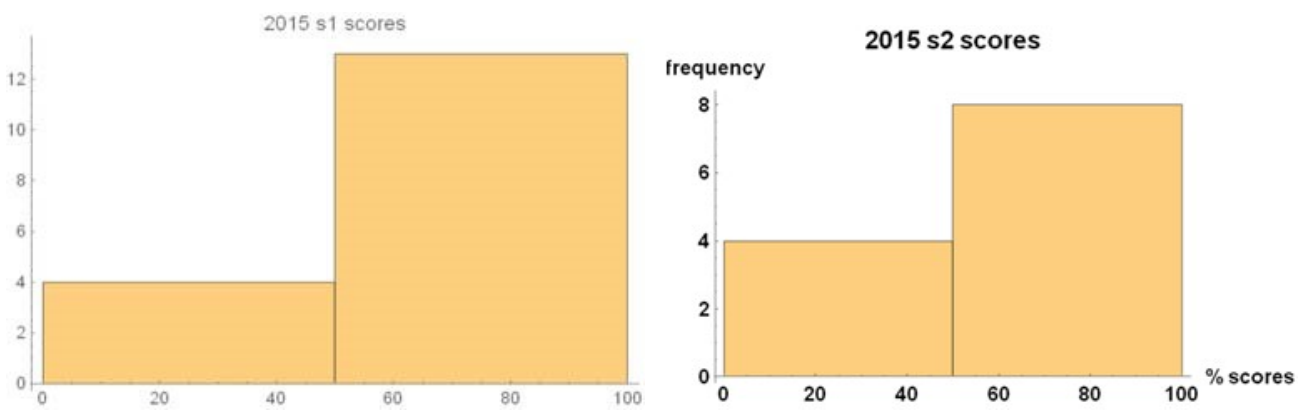

Figure 2. Assignment scores for S1 and S2 in 2015
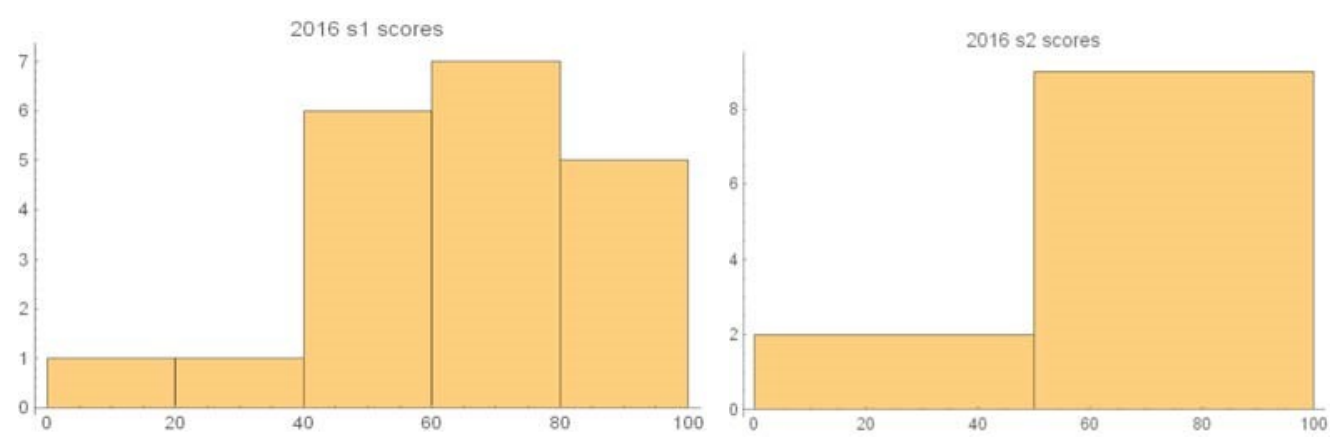

Figure 3. Assignment scores for S1 and S2 in 2016
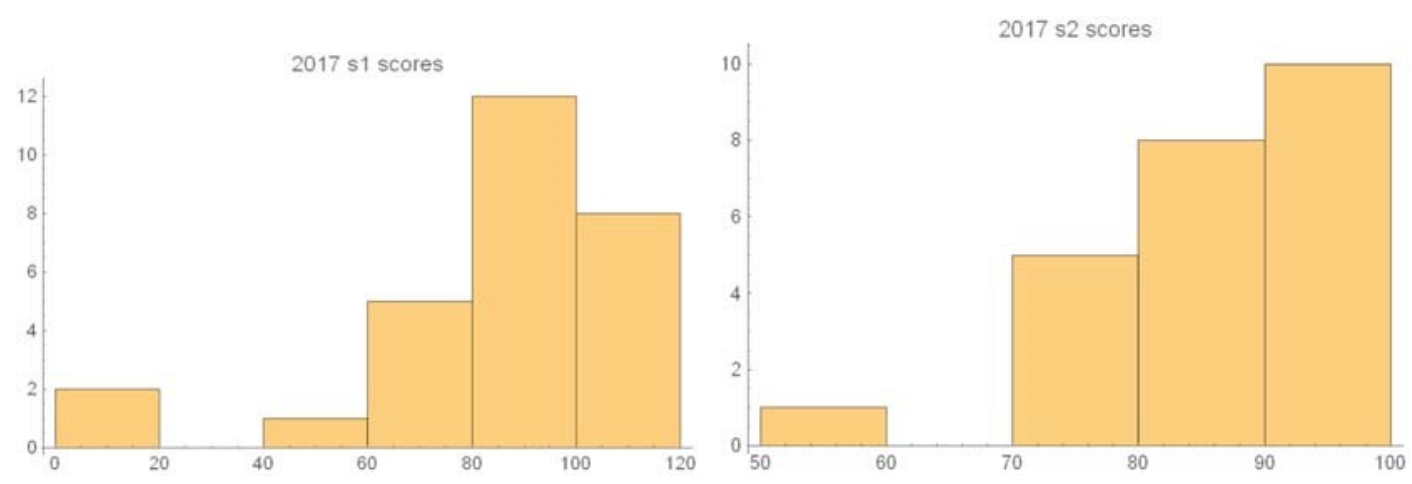

Figure 4. Assignment scores for S1 and S2 in 2017 


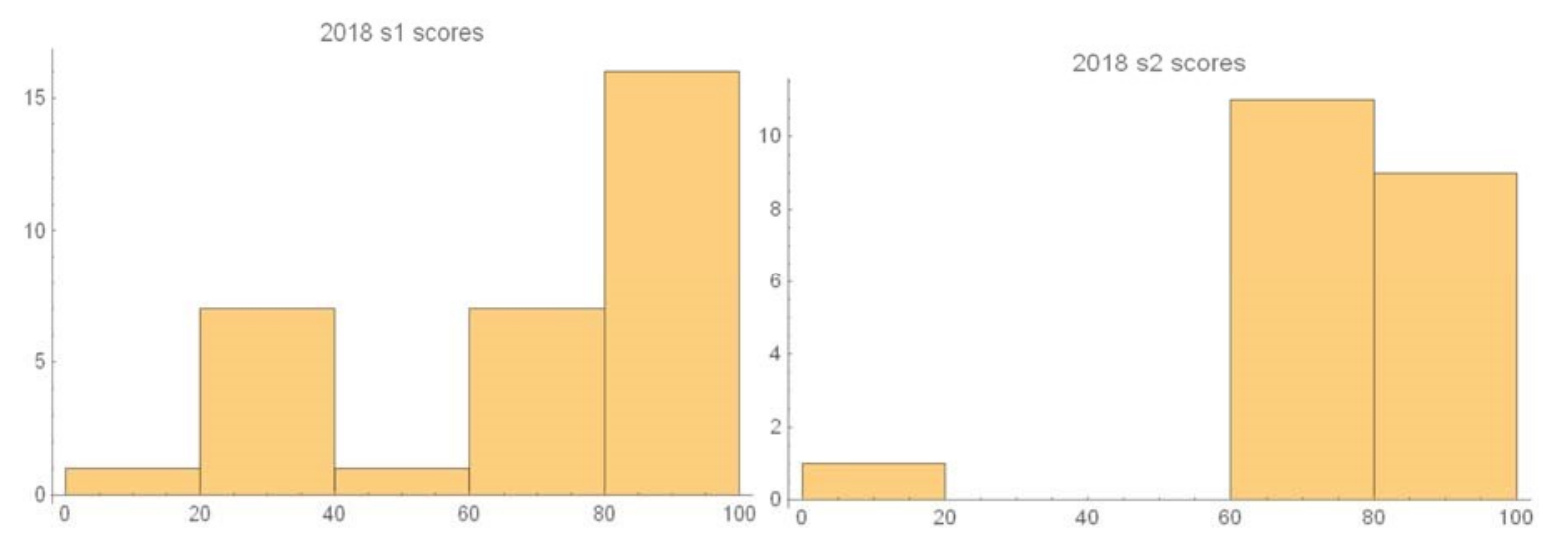

Figure 5. Assignment scores for S1 and S2 in 2018

In the beginning of the program there are clearly two homogenous groups of student, i.e. a big group of struggling students and another big group of good performance students. In the subsequent years, student performance began to stratify, and there are new groups formed, i.e. a small group of utterly struggling students and numerous groups of students with improved performance and a sizeable group of excelling students.

\section{Discussion}

An intervention of a teaching program which was instructionally designed to develop personalized and differentiated learning process for students, through breaking down lessons into lower and basic components, for struggling learners, and improves lessons to a complex high level and challenging activities for excelling students, proved to have produced positive results. First, the struggling students group is reduced gradually and the excelling students group is increased. Second, the two homogenous groups of good performance and struggling learners gradually disappears and they are replaced by numerous and smaller groups of learners performing above $50 \%$.

Towards the end of the study, i.e. 2017/2018, it becomes apparent that the group of excelling students increases and that of struggling students decrease significantly. It is also clear that more learners have improved their performance above 50\% and a large group is in the category of excellent performing students. The innovation introduced has improved student performance gradually and increased the number of excelling students. In general the student success rates has been increased and performance improved for a large number of learners for about $+25 \%$.

\section{Conclusion}

It is evident that this innovation integrates Moore's pedagogical variables, i.e. structure and dialogue, and builds a new conception for the four component of the intelligent tutor system (ITS), as inspired by Bloom's learning theory (Springer et al., 1999). The resultant is that student have an integrated view of the instructor's responsibilities (Petocz \& Reid, 
2003). They expect lecturers to be a catalyst for their learning and help them to change their view of the world of teaching and learning statistical content. Therefore, lecturers are seen to open students' minds to new possibilities and better performance.

This conception of teaching and learning is different from the common and general method, because it focuses on helping students to develop a higher level of comprehension of subject matter content. Therefore, the innovation implemented confirms that it is no more possible to treat all students in the proliferating range of e-learning users with very different prior knowledge, backgrounds, learning styles, interests and preferences, with the one-size-fits-all approach. It is thus, confirmed that when module contents is matched with the learning style of the learners along with considering the underlying pedagogical principles, learner's performance outcomes will improve, and therefore the hypothesis is confirmed.

\section{References}

Moore, M. G. (1991). Editorial: Distance education theory. The American Journal of Distance Education, 5(3), 1-6.

Moore, M. G. (1993). Theory of transactional distance. In D. Keegan (Ed.), Theoretical principles of distance education (pp. 22-38). New York: Routledge.

Moore, M. G., \& Kearsley, G. (1996). Distance Education: A systems view. New York: Wadsworth

Peters, O. (1994). Distance education and industrial production: A comparative interpretation in outline (1973). In D. Keegan (Ed.), Otto Peters on distance education: The industrialization of teaching and learning (p. 107-127). London: Routledge.

Petocz, P., \& Reid, A. (2003). Relationships between Students' Experience of Learning Statistics and Teaching Statistics. Statistics Education Research Journal, 2(1), 39-53. Retrieved from https://researchers.mq.edu.au/en/publications/relationships-betweenstudents-experience-of-learning-statistics-

Siadaty, M., \& Taghiyareh, F. (2007). PALS2: Pedagogically Adaptive Learning System based on Learning Style. Paper presented at the Seventh IEEE International Conference on Advanced Learning Technologies (ICALT 2007) 